\title{
Annual inspection of pressure vessel
}

\author{
Yannan $\mathrm{Du}^{1,2^{*}}$, Xuchen $\mathrm{Zhu}^{1}$, Bin Ren ${ }^{1,2}$, Xiaoying Tang ${ }^{1,2}$ \\ ${ }^{1}$ Shanghai Institute of Special Equipment Inspection and Technical Research, Putuo District, Shanghai, 200062, China \\ ${ }^{2}$ National Heat Exchanger Product Quality Inspection Center, Jinshan District, Shanghai, 201518, China
}

\begin{abstract}
This paper systematically discusses the significance of pressure vessel inspection, defines the main body of annual inspection of pressure vessel, and puts forward the matters needing attention in the process of annual inspection according to the existing annual inspection situation, so as to provide a certain reference for the smooth development of annual inspection of pressure vessels.
\end{abstract}

\section{Introduction}

As eight kinds of special equipment, the safety of fixed pressure vessel has been widely valued by the whole society. However, there are obvious differences in the degree of familiarity and mastery of special equipment laws and regulations[1-3] among enterprise safety management personnel. Due to the legal inspection, most enterprises could carry out regular inspection on time. The annual inspection work of regular self-inspection is often ignored, even confused with daily inspection and regular inspection. In addition, due to the limitation of the technical level of the pressure vessel professionals, it is difficult to carry out the annual self-inspection of pressure vessels. Due to the existence of a certain inspection cycle in the periodic inspection and the lack of necessary annual inspection work in the inspection cycle of pressure vessels, there may be certain potential safety hazards and serious consequences[4-6]. This paper combs the annual inspection of pressure vessels, analyzes and discusses some problems involved in the annual inspection of pressure vessels, so as to provide certain reference for the relevant provisions of annual inspection of pressure vessels.

\section{Annual inspection of pressure vessel}

\subsection{Significance of annual inspection}

The annual inspection of in-service stationary pressure vessels (hereinafter referred to as pressure vessels) is not a statutory inspection. However, in order to ensure the safe operation of pressure vessels within the inspection cycle, the annual inspection is also a necessary inspection work, which is an important regular selfinspection to ensure the safety of special equipment, In the special equipment safety law, TSG 21-2016 "Supervision Regulation on Safety Technology for Stationary Pressure Vessel", TSG 08-2017 " Special
Equipment Service Administration Regulation", there are relevant fixed. Article 39 of the "special equipment safety law" clearly stipulates that the unit using special equipment shall carry out regular maintenance and regular self-inspection of the special equipment it uses, and make records.

According to Section 2.2 of TSG 08-2017 technical specification for safety of special equipment, the main obligation of the user is that the user shall carry out regular maintenance and regular self-inspection on the special equipment in use, and timely check and eliminate potential safety hazards. TSG 21-2016, Chapter 7 stipulates that the user of pressure vessels shall conduct regular self-inspection, including monthly inspection and annual inspection. The annual inspection shall be conducted at least once a year. After the annual inspection, the use safety status shall be analyzed, and the hidden dangers found in the annual inspection shall be eliminated in time.

If the enterprise fails to carry out the annual inspection, the special equipment supervision personnel may, according to the provisions of Article 83, paragraph 3 of the special equipment safety law, impose a fine of not less than $¥ 10,000$ but not more than $¥ 100,000$ on the using unit.According to TSG 21-20168.7.1, the inspection unit of pressure vessel can also shorten the periodic inspection cycle appropriately. In addition to shortening the annual inspection period, it will also cause the inspection and inspection of the enterprise.

\subsection{Main object of annual inspectionl}

The annual inspection of pressure vessels can be carried out by specially trained operators organized by the safety management personnel of the pressure vessel using unit, or by a qualified special equipment inspection institution. It could be seen that the main object of annual inspection is the user.

The meaning of user is clearly defined in Section 2.1 of TSG $08-2017$. The user list mainly refers to the unit with the right to use and manage special equipment

*Corresponding author's e-mail:ynduzgz@163.com 
(including companies, subsidiaries, institutions, social organizations and other units with legal personality, branches with business license, individual businesses, etc.) or natural persons with full civil capacity, Generally, it is the property right unit of special equipment (property owner, the same below), and it can also be the actual use manager of special equipment established by the property right unit through the contractual relationship in accordance with the law. Special equipment belongs to the common ownership, the coowner may entrust the property service unit or other managers to manage the special equipment, and the trustee is the user unit; if the coowner does not entrust, the actual manager is the user unit; if there is no actual manager, the coowner is the user unit. If special equipment is used for leasing, the leasing unit shall be the user during the lease period; if there are other provisions in the law or in the contract between the parties, such provisions or agreements shall prevail.

\section{Annual inspection and precautions}

\subsection{Basis for annual inspection}

The annual inspection items shall at least include the safety management of pressure vessels, the body and operation of pressure vessels and the safety accessories of pressure vessels, especially the inspection of safety accessories and instruments, including the inspection of safety valves, bursting disc devices, safety interlock devices, etc., and the inspection of instruments includes the inspection of pressure gauges, liquid level meters, temperature measuring instruments, etc.In the regular inspection items, in addition to the safety valve, bursting disc and safety interlock device, other safety accessories and instruments are no longer required inspection items, and more attention should be paid in daily inspection.

\subsection{Annual inspection}

The specific inspection contents and inspection requirements of the annual inspection of pressure vessels are described in detail in section 7.2 of the code, and each inspection content covers specific inspection items. However, based on the inspection results of our hospital and the reports of other units, the overall work quality of the annual inspection of pressure vessels is not high, and the detection rate of hidden dangers is low except for the overdue use of safety accessories.

\subsubsection{Safety management of pressure vessels}

All pressure vessels are involved in the code. Enterprises need to formulate special annual inspection procedures according to the production characteristics, process principle and equipment type of the enterprise. Combined with the actual inspection and the literature reports, at present, the annual inspection work of quite a few enterprises is just a formality, which is not applicable to the characteristics of pressure vessels of their own units, and the on-site inspection items are missing, Even there are no items of inspection (such as the inspection of cryogenic tank blowdown), which has lost the significance of annual inspection to prevent accidents.

\subsubsection{Pressure vessel body and operation condition inspection}

(1) Inspection of leak detection hole and signal hole. Leakage indication holes are required on the reinforcing ring and reinforcing base plate of pressure vessels, and the leakage signal indicating holes should be kept smooth. In the actual process, most of the leak detection holes are blocked by paint, and the leak detection holes and signal holes lack the installation function, which is also the blind area in the inspection process.

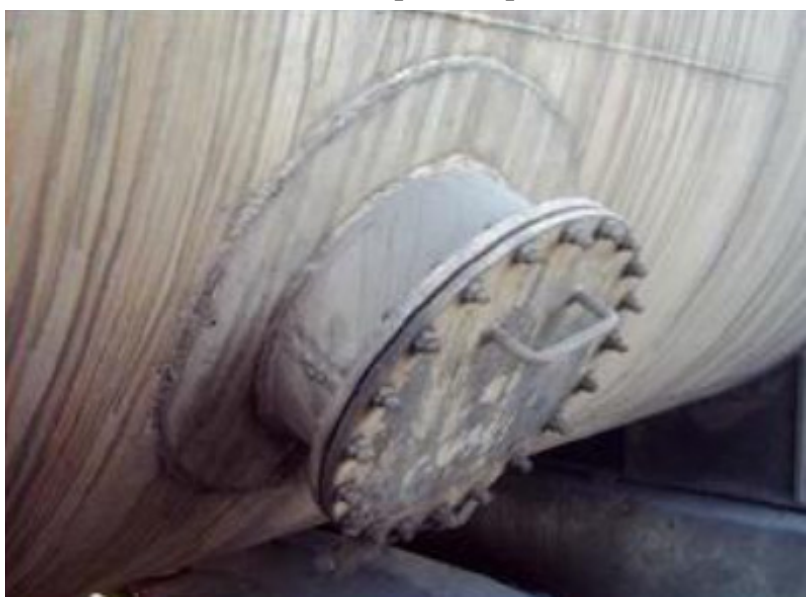

Figure 1. Reinforcement Pad

(2) Inspection of drainage (drainage and sewage) devices. The blowdown device is hardly checked. If the operator should be required to manually operate the blowdown valve to confirm whether the valve can operate normally. At the same time, the cryogenic equipment generally has no blowdown device. During the annual inspection of each unit, there are no items checked, which makes the inspection meaningless.

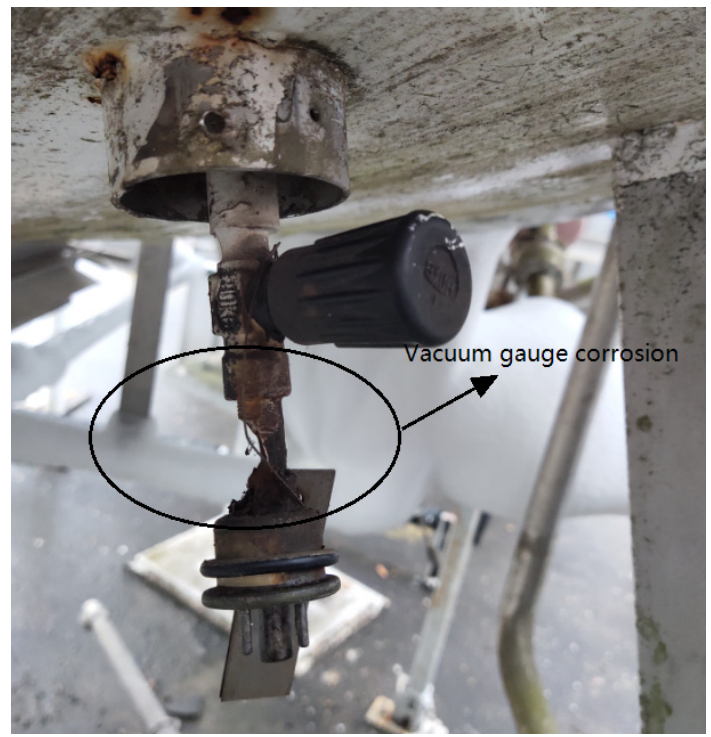

Figure 2. Vacuum gauge corrosion 
(3) Inspection of tank body with grounding device. If there is no grounding device for the liquid liquid or the oil tank, check whether it meets the requirements.

(4) Medium monitoring. For example, the purity of ammonia in the medium will be limited when the ammonia storage vessel leaves the factory to prevent ammonia stress corrosion cracking. The purer the ammonia is, the better it is. Flash-steams-tank uses water to generate steam. Unqualified water quality may cause abnormal corrosion of equipment, but in the actual inspection process, the project is rarely covered.

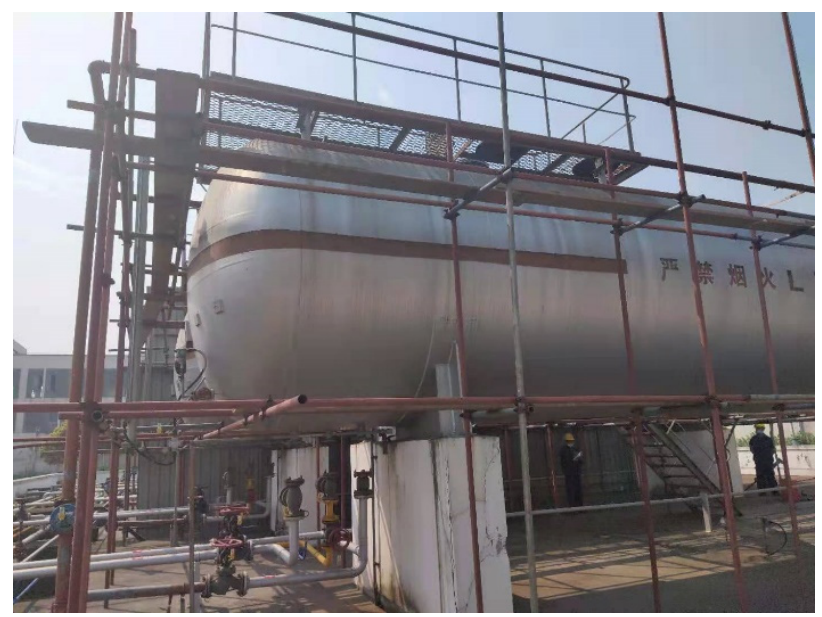

Figure 3. LPG Storage Tanks

\subsubsection{Inspection of safety accessories and instruments of pressure vessels}

(1) Safety valve inspection. Whether the discharge capacity of the safety valve meets the requirements mainly depends on checking the design data or previous inspection reports. However, in the actual inspection process, due to the lack of original factory data and inspection report, more attention is paid to whether the set pressure is qualified and whether the verification cycle is overdue, and the selection and material of safety valve are ignored. For example, for pressure vessels containing toxic and explosive media, the stop valve is installed between the safety valve and the pressure vessel, and the discharge port is not installed with lead seal or lock, and the discharge port is not installed with a conduit, Lead the discharged medium to a safe place. The setting pressure value of safety valve is low. The setting pressure value of safety valve is calculated according to the working pressure required by production process. It is considered that the lower the set pressure is, the safer it is. In fact, this is a wrong understanding. The function of safety valve is mainly used to protect pressure vessels.

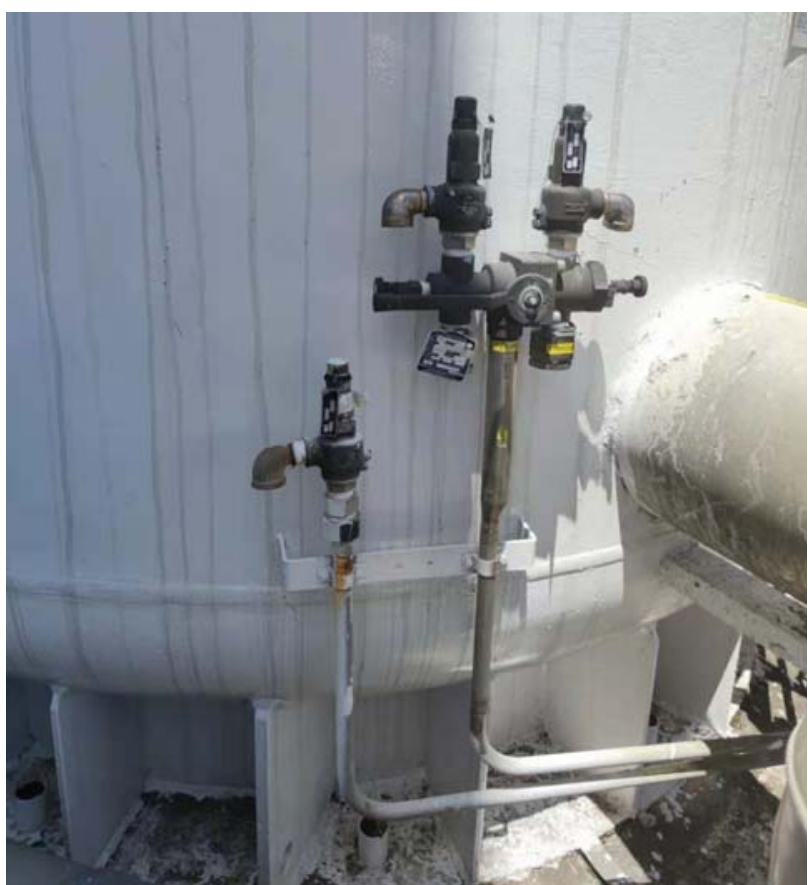

Figure 4. Safety valve and Rupture Disc of LPG Storage Tanks

(2) Inspection of bursting disc device. It is necessary to check the ex factory data of bursting discs, check whether the manufacturer has the corresponding special equipment manufacturing license, and whether the bursting pressure of bursting discs meets the requirements. During the inspection process, there are some containers which are difficult to be inspected due to the loss of factory data. The inspection personnel rely on experience and the information provided by the manufacturer for the bursting discs, which may be missed for inspection and replacement.

(3) Safety interlock. Check whether the safety interlock device has been installed on the quick opening pressure vessel, and whether the function of the safety interlock device in use meets the relevant requirements and can be used normally.

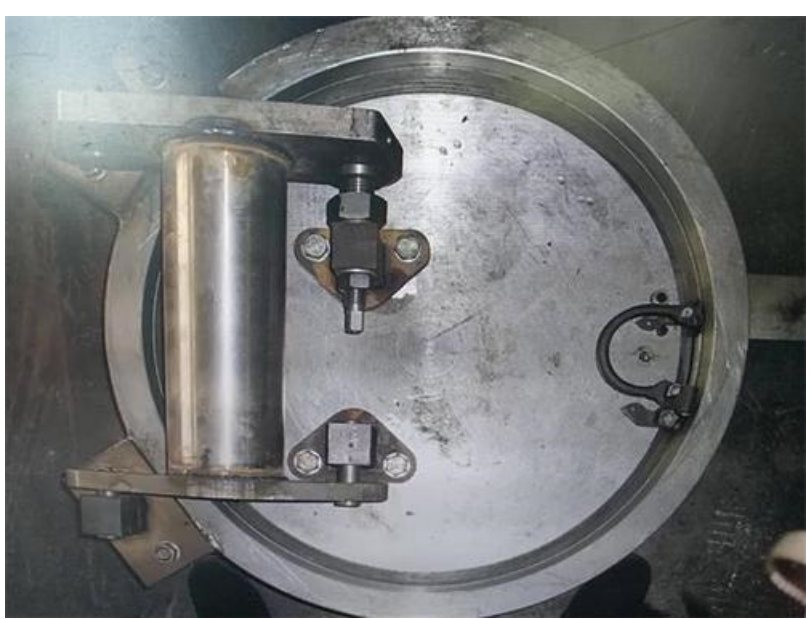

Figure 5. Safety Interlock Unit

(4) Pressure gauge inspection. Pay more attention to whether the range meets the range, and less check whether the selected pressure gauge is suitable for the 
medium of pressure vessel. For example, the pressure gauge of liquid ammonia storage tank adopts non ammonia pressure gauge, and copper is forbidden to be used in ammonia medium (except phosphorus bronze).For the pressure gauge used in the steam water environment, whether the water trap is installed between the pressure gauge and the pressure vessel.

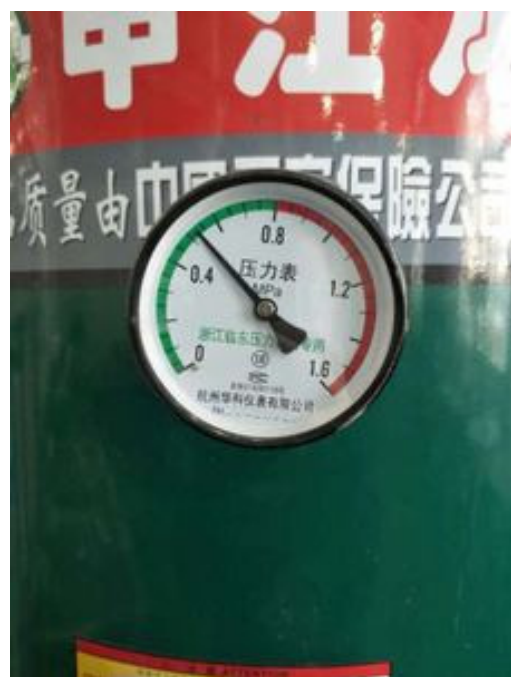

Figure 6. Pressure gauge

(5) Temperature measuring instrument inspection. In the inspection, it is often neglected to check whether there is regular maintenance and verification.

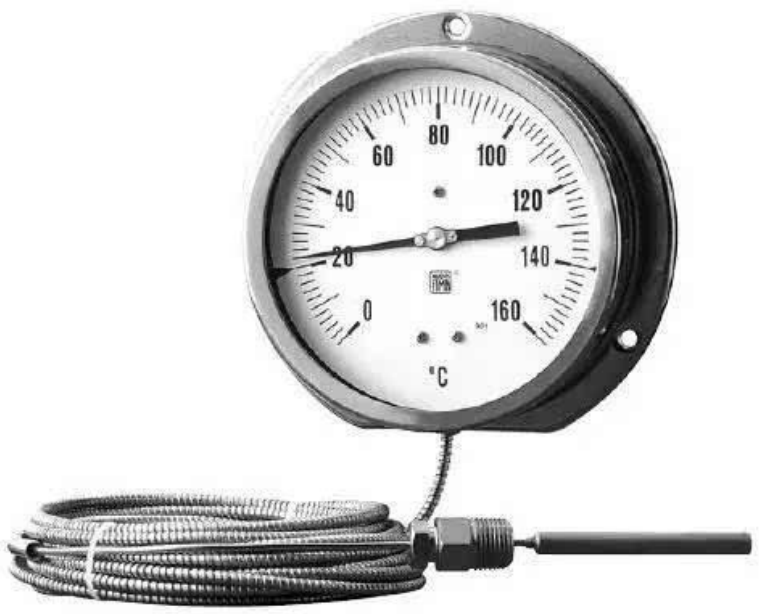

Figure 7. Temperature Measuring Instrument

(6) Level gauge inspection. In the process of inspection, the regular maintenance system of the level gauge and the hydraulic test record before installation and use are often neglected, and whether the maximum and minimum safe liquid level on the level gauge are marked with obvious marks.

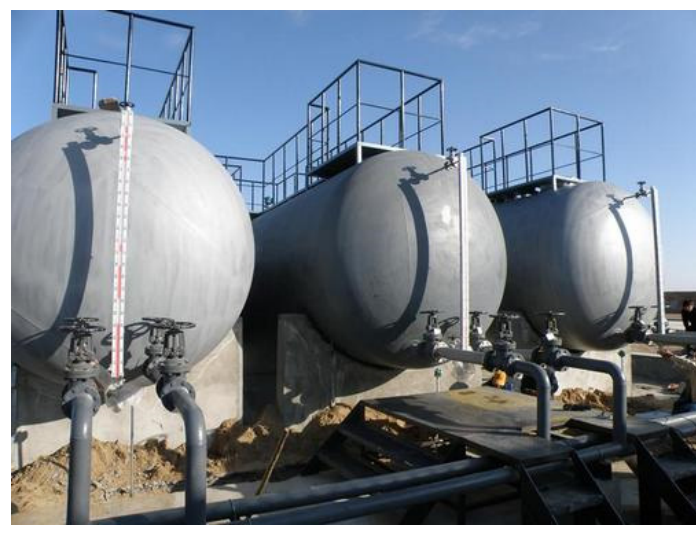

Figure 8. Level Gauge Inspection

\section{Conclusions}

This paper systematically discusses the significance of pressure vessel inspection, defines the main body of annual inspection of pressure vessel, and puts forward the matters needing attention in the process of annual inspection according to the existing annual inspection situation. In view of the blind areas and deficiencies in the annual inspection of pressure vessels, relevant personnel should strengthen the study of relevant laws and regulations on pressure vessels, Further improve the effect of annual inspection of pressure vessels to ensure the safe operation of pressure vessels in the inspection cycle.

\section{Acknowledgments}

This study has received funding by Scientific Research Projects of Shanghai Quality and Technical Supervision 2019-22 and 2019-25, and Shanghai Science and Technology Talents Program (19XD1432600). The authors are grateful for the financial supports provided by Shanghai engineering research center of pressure pipeline intelligent inspection and National Heat Exchanger Product Quality Inspection Center.

\section{References}

1. Special Equipment Safety Law of PRC[S]. 2013.

2. TSG 21-2016. Supervision Regulation on Safety Technology for Stationary Pressure Vessel [S]. 2016.

3. TSG 08-2017. Supervision Regulation on Safety Technology for Stationary Pressure Vessel [S]. 2016.

4. Ma, CB.(2020). Significance of annual inspection of pressure vessels. Encyclopedia Form. (9):1498.

5. Jiang, C., Lin, XS., Zeng, DC., (2011) Pressure Vessels'overall inspection and annual inspection. China Science \& Technology Overview. (17):278-278.

6. Zhao, WL., (2020). Annual inspection and perfection management inspection strategy of special equipment. In The second symposium on corrosion and safety in petrochemical industry. 2016:173-175. 